\title{
Approximate Controllability for Functional Equations with Riemann-Liouville Derivative by Iterative and Approximate Method
}

\author{
Badawi Hamza Elbadawi Ibrahim, Zhenbin Fan, and Gang Li \\ School of Mathematical Sciences, Yangzhou University, Yangzhou, Jiangsu 225002, China \\ Correspondence should be addressed to Gang Li; gli@yzu.edu.cn
}

Received 31 March 2017; Accepted 28 May 2017; Published 27 June 2017

Academic Editor: Lishan Liu

Copyright (C) 2017 Badawi Hamza Elbadawi Ibrahim et al. This is an open access article distributed under the Creative Commons Attribution License, which permits unrestricted use, distribution, and reproduction in any medium, provided the original work is properly cited.

\begin{abstract}
We discuss the functional control systems governed by differential equations with Riemann-Liouville fractional derivative in general Banach spaces in the present paper. First we derive the uniqueness and existence of mild solutions for functional differential equations by the approach of fixed point and fractional resolvent under more general settings. Then we present new sufficient conditions for approximate controllability of functional control system by means of the iterative and approximate method. Our results unify and generalize some previous works on this topic.
\end{abstract}

\section{Introduction}

As we all know, the study of fractional calculus theory can be traced back to the end of the seventeenth century. In recent years, a considerable interest has been paid to functional evolution equations with fractional derivatives since they are of importance in describing the natural phenomenon including the models in stochastic processes, finance, and physics (see [1-9]). On the other hand, the notion of controllability plays a central role in the study of the theory of control and optimization. Therefore, there are a lot of works on the controllability, approximate controllability, and optimal control of linear and nonlinear differential and integral systems in various frameworks (see [10-28] and references therein).

Recently, the theory of resolvent families was formulated rapidly for the application of differential and integral equations, including the concepts of integrated solution operators [29], fractional resolvent operators [30], and $(a, k)$ regularized resolvent operators [19]. Furthermore, there are extensive studies for the control systems governed by Caputo fractional evolution equations via resolvent theory (see, for instance, $[10-12,15,27,31])$. However, for the controllability of functional differential systems governed by RiemannLiouville fractional derivatives there are few results to be shown so far.

In $[32,33]$, the authors established the general theory of fractional resolvent and studied its application to the well posed problem for the evolution equation below:

$$
\begin{aligned}
D^{\beta} y(t) & =A y(t)+g(t), t \in(0, c], \\
\lim _{t \downarrow 0} \Gamma(\beta) t^{1-\beta} y(t) & =y \in X,
\end{aligned}
$$

where $D^{\beta}$ is the standard Riemann-Liouville derivative with $0<\beta \leq 1$ and operator $A: D(A) \subset X \rightarrow X$ generates a $\beta$-order fractional resolvent $S_{\beta}(t)$. On the other hand, in [17], the authors considered the following control system governed by functional differential equations with Riemann-Liouville derivative in an abstract space $X$ :

$$
\begin{array}{r}
D^{\beta} y(t)=A y(t)+B u(t)+g(t, y(t)), \\
t \in(0, c],
\end{array}
$$

$\lim _{t \downarrow 0} \Gamma(\beta) t^{1-\beta} y(t)=y_{0} \in X$ 
where $D^{\beta}$ is the standard Riemann-Liouville derivative with $0<\beta \leq 1$ and operator $A: D(A) \subset X \rightarrow X$ generates a semigroup $S(t), t \geq 0$, on an abstract space $X . B: V \doteq L^{p}([0, c], U) \rightarrow L^{p}([0, c], X)$ belongs to the space $\mathbf{B}\left(V, L^{p}([0, c], X)\right), U$ is a Banach space, and the control function $u$ belongs to $V$.

Motivated by the above-mentioned papers, we try to solve the approximate controllability for functional differential equation (2) again but under the assumption that operator $A$ : $D(A) \subset X \rightarrow X$ is the infinitesimal generator of a resolvent $S_{\beta}(t), t>0$, on the general Banach space $X$. Under this general condition, the difficulty on the well-posedness is how to deal with the singularity of resolvent operators and solutions at zero. We deal with this problem by utilizing the new space $C_{1-\beta}([0, c], X)$ and the theory of fractional resolvent developed in $[32,33]$. In the present article, we first obtain the uniqueness and existence of mild solutions for functional differential equation (2) via fractional resolvent and topological approach. Then we will give the sufficient conditions for approximate controllability to equation (2) by means of the iterative and approximate method. Our main result seems to be more general and extends some recent related theorems.

In this paper, we first review some basic definitions and give some necessary lemmas. We establish the uniqueness and existence results of mild solutions for functional differential equation (2) in the third part. We will solve the approximately controllable problem of functional control system (2) in the last section.

\section{Preliminaries}

This section is devoted to introducing some necessary concepts and auxiliary results which are used in the remainder of this article.

Let $X, Y$ be two Banach spaces with norm $\|\cdot\|$ and $\mathbf{R}_{+}$be the set of nonnegative real numbers. We denote by $\mathbf{B}(X)$ all the continuous linear operators from Banach space $X$ into itself, by $C([0, c], X)$ the set of all the continuous functions from the interval $[0, c]$ to Banach space $X$ with $\|y\|_{C}=\sup \{\|y(t)\|, t \in[0, c]\}$, and by $L^{P}([0, c], X)$ the set of all Bochner integrable functions from the interval $[0, c]$ to Banach space $X$ with $\|g\|_{L^{p}}=\left(\int_{0}^{c}\|g(\tau)\|^{p} d \tau\right)^{1 / p}$, where $1 \leq p<\infty$.

Let $A C([0, c], X)$ be the set of all absolutely continuous functions from the interval $[0, c]$ to Banach space $X$, and $A C^{m}([0, c], X)=\left\{g:[0, c] \rightarrow X\right.$ and $g^{(m-1)} \in$ $A C([0, c], X)\}$. Let $0<\beta \leq 1$; we consider the Banach space

$$
C_{1-\beta}([0, c], X)=\left\{y: \tau^{1-\beta} y(\tau)\right.
$$

is continuous on the interval $[0, c]$

$$
\text { with values in } X\}
$$

with $\|y\|_{C_{1-\beta}}=\sup \left\{\tau^{1-\beta}\|y(\tau)\|: \tau \in[0, c]\right\}$. This space is of importance in dealing with the well-posedness of the functional equation (2).
As usual, let $\Gamma(z) \doteq \int_{0}^{\infty} e^{-\tau} \tau^{z-1} d \tau$ denote the Gamma function and $[\beta]$ the integer part of the real number $\beta>0$. We give the following definitions.

Definition 1 (see $[3,34]$ ). The Riemann-Liouville fractional integral of order $\beta>0$ for a function $h \in L^{1}([0, c], X)$ is defined by

$$
I_{t}^{\beta} h(t)=\frac{1}{\Gamma(\beta)} \int_{0}^{t} \frac{h(\tau)}{(t-\tau)^{1-\beta}} d \tau, \quad 0 \leq t \leq c,
$$

if the integral above exists point-wisely.

Definition 2 (see $[3,34]$ ). Let $m=[\beta]+1$. The RiemannLiouville fractional derivative of order $\beta>0$ for a function $h \in L^{1}([0, c], X)$ is defined by

$$
\begin{aligned}
D^{\beta} h(t)=\frac{1}{\Gamma(m-\beta)} \frac{d^{m}}{d t^{m}} \int_{0}^{t} \frac{h(\tau)}{(t-\tau)^{\beta+1-m}} d \tau, & \\
& 0 \leq t \leq c,
\end{aligned}
$$

if the derivative of $m$-order above exists point-wisely.

Lemma 3 (see $[3,34])$. Let $m=[\beta]+1$ and $y_{m-\beta}(t)=$ $I_{t}^{m-\beta} y(t)$. Suppose $y(\cdot) \in L^{1}([0, c], X)$ and $y_{m-\beta}(\cdot) \in$ $A C^{m}([0, c], X)$. Then, one has

$$
I_{t}^{\beta} D^{\beta} y(t)=y(t)-\sum_{k=1}^{m} \frac{y_{m-\beta}^{(m-k)}(0)}{\Gamma(\beta-k+1)} t^{\beta-k}, \quad 0 \leq t \leq c .
$$

Definition 4 ([32, Definition 3.1]). Let $0<\beta \leq 1$. A family of operators $\left\{S_{\beta}(t)\right\}_{t>0}$ belonging to the space $\mathbf{B}(X)$ is called a $\beta$-order fractional resolvent if

(a) $S_{\beta}(t)$ is continuous in the strong operator topology on $[0, \infty)$, and $\lim _{\tau \downarrow 0}\left(\left(S_{\beta}(\tau)\right) / \tau^{\beta-1}\right) y=(1 / \Gamma(\beta)) y$ for $y \in X$.

(b) $S_{\beta}(\mu) S_{\beta}(\tau)=S_{\beta}(\tau) S_{\beta}(\mu)$ for any $\mu, \tau>0$.

(c) $S_{\beta}(\mu) I_{\tau}^{\beta} S_{\beta}(\tau)-I_{\mu}^{\beta} S_{\beta}(\mu) S_{\beta}(\tau)=\left(\mu^{\beta-1} / \Gamma(\beta)\right) I_{\tau}^{\beta} S_{\beta}(\tau)-$ $\left(\tau^{\beta-1} / \Gamma(\beta)\right) I_{\mu}^{\beta} S_{\beta}(\mu)$ for all $\mu, \tau>0$.

Definition 5. The infinitesimal generator $A$ of the fractional resolvent $\left\{S_{\beta}(t)\right\}_{t>0}$ is defined by

$$
A y=\Gamma(2 \beta) \lim _{\tau \downarrow 0} \frac{S_{\beta}(\tau) y-\left(\tau^{\beta-1} / \Gamma(\beta)\right) y}{\tau^{2 \beta-1}}
$$

with

$$
\begin{aligned}
& D(A) \\
& \quad=\left\{y \in X: \lim _{\tau \downarrow 0} \frac{S_{\beta}(\tau) y-\left(\tau^{\beta-1} / \Gamma(\beta)\right) y}{\tau^{2 \beta-1}} \text { exists }\right\} .
\end{aligned}
$$

Lemma 6 ([32, Theorem 3.2]). Let $A$ be the infinitesimal generator of the fractional resolvent $\left\{S_{\beta}(t)\right\}_{t>0}$ on Banach space $X$. Then $A$ is closed with dense domain and the following hold. 
(a) $S_{\beta}(\tau) D(A) \subset D(A)$ and $A S_{\beta}(\tau) y=S_{\beta}(\tau) A y$ for $y \epsilon$ $D(A), \tau>0$.

(b) For $\tau>0$, one has

$$
\begin{aligned}
& S_{\beta}(\tau) y=\frac{\tau^{\beta-1}}{\Gamma(\beta)} y+A I_{\tau}^{\beta} S_{\beta}(\tau) y, \quad y \in X, \\
& S_{\beta}(\tau) y=\frac{\tau^{\beta-1}}{\Gamma(\beta)} y+I_{\tau}^{\beta} S_{\beta}(\tau) A y, \quad y \in D(A) .
\end{aligned}
$$

Lemma 7. Let $c>0$ be fixed and operator $A$ be the generator of a $\beta$-order fractional resolvent $\left\{S_{\beta}(t)\right\}_{t>0}$ in Banach space $X$ and

$$
\left.\tau^{1-\beta} S_{\beta}(\tau) y\right|_{\tau=0} \doteq \lim _{\tau \downarrow 0} \tau^{1-\beta} S_{\beta}(\tau) y=\frac{1}{\Gamma(\beta)} y,
$$

$$
y \in X
$$

Then there is a positive number $M>0$ satisfying $\sup _{\tau \in[0, c]}\left\|\tau^{1-\beta} S_{\beta}(\tau)\right\| \leq M$.

Proof. According to the definition of $\beta$-order fractional resolvent, for every $y \in X$ there is a positive number $M_{y}>0$ dependent on $y$ satisfying

$$
\left\|\tau^{1-\beta} S_{\beta}(\tau) y\right\| \leq M_{y}, \quad \forall \tau \in[0, c] .
$$

Moreover, since $\tau^{1-\beta} S_{\beta}(\tau) \in \mathbf{B}(X), \tau \in[0, c]$, it follows from the uniform boundedness principle that there is a positive number $M>0$ independent of $y$ satisfying $\sup _{\tau \in[0, c]}\left\|\tau^{1-\beta} S_{\beta}(\tau)\right\| \leq M$.

Now, we turn to the concept of mild solutions and approximate controllability of functional equation (2). In [33], Fan proved that the convolution $S_{\beta} * g$ exists and defines a continuous function on $[0, c]$ when the resolvent is uniformly integrable and the function $f$ is continuous on $[0, c]$. In fact, by the Proposition 1.3.4 in [35] and the Young inequality, we can also prove that the above convolution exists and defines a continuous function on $[0, c]$ under the assumption that $S_{\beta}(t)$ is just a fractional resolvent and $g \in$ $L^{1}([0, c], X)$. Thus, we have the following definitions.

Definition 8. A mapping $y(\cdot) \in C_{1-\beta}([0, c], X)$ is called a mild solution of functional differential equation (2) if for $u \in V$, the integral equation

$$
\begin{aligned}
y(t)= & S_{\beta}(t) y_{0} \\
& +\int_{0}^{t} S_{\beta}(t-\tau)[B u(\tau)+g(\tau, y(\tau))] d \tau, \\
& 0<t \leq c
\end{aligned}
$$

is satisfied.

Let $y\left(\cdot ; 0, y_{0}, u\right)$ be a mild solution of functional equation (2) with the control function $u$ belonging to the space $V$ and initial value $y_{0}$ in Banach space $X$. Define the reachable set of functional equation (2) at time $c$ by $K\left(c, y_{0}\right) \doteq\left\{y\left(c ; 0, y_{0}, u\right)\right.$ : $u \in V\}$.
Definition 9. The functional equation (2) is called approximate controllability on $[0, c]$ if $\overline{K\left(c, y_{0}\right)}=X$.

\section{The Result of Uniqueness and Existence for Mild Solutions}

In this part, we study the existence and uniqueness of solutions for functional equation (2). To prove our result, we suppose the following conditions.

(H1) $A$ is the infinitesimal generator of an analytic $\beta$ order fractional resolvent of continuous linear operators $\left\{S_{\beta}(t)\right\}_{t>0}$ on Banach space $X$.

(H2) There are nonnegative function $\mu(\cdot) \in L^{p}\left([0, c], \mathbf{R}_{+}\right)$ and a real number $k>0$ satisfying

$$
\|g(\tau, y)\| \leq \mu(\tau)+k \tau^{1-\beta}\|y\|
$$

for $y \in X$ and $\tau \in[0, c]$.

(H3) There is a nonnegative real number $L$ satisfying

$$
\|g(\tau, y)-g(\tau, z)\| \leq L\|y-z\|
$$

for $\tau \in[0, c]$ and $y, z \in X$.

Now, we can give the existence result of mild solutions for the functional equation (2).

Theorem 10. Under the conditions (H1)-(H3), the functional equation (2) has one and only one mild solution in the space $C_{1-\beta}([0, c], X)$ for every $u$ belonging to the control space $V$.

Proof. Define the mapping $Q: C_{1-\beta}([0, c], X) \rightarrow C_{1-\beta}([0$, c], $X)$ by

$$
\begin{aligned}
(\mathrm{Q} y)(t)=S_{\beta}(t) y_{0} & \\
& +\int_{0}^{t} S_{\beta}(t-\tau)[B u(\tau)+g(\tau, y(\tau))] d \tau, \\
& 0<t \leq c .
\end{aligned}
$$

Obviously, it is well-defined. To prove our result, it is enough to verify the mapping $Q$ has a unique fixed point in space $C_{1-\beta}([0, c], X)$. We next verify that $Q^{n}$ is a contraction map on $C_{1-\beta}([0, c], X)$ for sufficiently large integer number $n$.

According to Lemma 3 , there is a constant $M>0$ satisfying $\sup _{\tau \in[0, c]}\left\|\tau^{1-\beta} S_{\beta}(\tau)\right\| \leq M$. Thus, for any $y, z \in C_{1-\beta}([0$, $c], X)$ and $0 \leq t \leq c$, one has

$$
\begin{aligned}
t^{1-\beta} & \|(Q y)(t)-(Q z)(t)\| \leq t^{1-\beta} \int_{0}^{t}(t-\tau)^{\beta-1} \\
\cdot & \left\|(t-\tau)^{1-\beta} S_{\beta}(t-\tau)\right\| \\
\cdot & \|g(\tau, y(\tau))-g(\tau, z(\tau))\| d \tau \\
& \leq t^{1-\beta} \int_{0}^{t}(t-\tau)^{\beta-1} \tau^{\beta-1}\left\|(t-\tau)^{1-\beta} S_{\beta}(t-\tau)\right\| \\
& \cdot \tau^{1-\beta} L\|y(\tau)-z(\tau)\| d \tau
\end{aligned}
$$




$$
\begin{aligned}
& \leq t^{1-\beta} M L \int_{0}^{t}(t-\tau)^{\beta-1} \tau^{\beta-1}\|y-z\|_{C_{1-\beta}} d \tau \\
& =\frac{t^{\beta} M L[\Gamma(\beta)]^{2}}{\Gamma(2 \beta)}\|y-z\|_{C_{1-\beta}} .
\end{aligned}
$$

Further,

$$
\begin{aligned}
& t^{1-\beta}\left\|\left(Q^{2} y\right)(t)-\left(Q^{2} z\right)(t)\right\| \leq t^{1-\beta} M L \int_{0}^{t}(t-\tau)^{\beta-1} \\
& \cdot \tau^{\beta-1} \tau^{1-\beta}\|(Q y)(\tau)-(Q z)(\tau)\| d \tau \\
& \leq t^{1-\beta} M L \int_{0}^{t}(t-\tau)^{\beta-1} \tau^{\beta-1} \\
& \cdot \frac{M L[\Gamma(\beta)]^{2}}{\Gamma(2 \beta)} \tau^{\beta}\|y-z\|_{C_{1-\beta}} d \tau \\
& \leq t^{1-\beta} M L \int_{0}^{t}(t-\tau)^{\beta-1} \tau^{2 \beta-1} \\
& \cdot \frac{M L[\Gamma(\beta)]^{2}}{\Gamma(2 \beta)}\|y-z\|_{C_{1-\beta}} d \tau \\
& =\frac{\left(t^{\beta} M L\right)^{2}[\Gamma(\beta)]^{3}}{\Gamma(3 \beta)}\|y-z\|_{C_{1-\beta}} .
\end{aligned}
$$

By repeating the above process, one has

$$
\begin{aligned}
& t^{1-\beta}\left\|\left(Q^{n} y\right)(t)-\left(Q^{n} z\right)(t)\right\| \\
& \quad \leq \frac{\left(c^{\beta} M L\right)^{n}[\Gamma(\beta)]^{n+1}}{[\Gamma(n+1) \beta]}\|y-z\|_{C_{1-\beta}} .
\end{aligned}
$$

Thus,

$$
\left\|Q^{n} y-Q^{n} z\right\|_{C_{1-\beta}} \leq \frac{\Gamma(\beta)\left(c^{\beta} M L \Gamma(\beta)\right)^{n}}{[\Gamma(n+1) \beta]}\|y-z\|_{C_{1-\beta}} .
$$

Note that the Mittag-Leffler function $E_{\beta, \beta}\left(c^{\beta} M L \Gamma(\beta)\right)=$ $\sum_{k=0}^{\infty}\left(\left(c^{\beta} M L \Gamma(\beta)\right)^{k} / \Gamma(k \beta+\beta)\right)$ is uniformly convergent; one has sufficiently large integer $n$,

$$
\frac{\Gamma(\beta)\left(c^{\beta} M L \Gamma(\beta)\right)^{n}}{[\Gamma(n+1) \beta]}<1,
$$

which implies $Q^{n}$ is a contraction map on the space $C_{1-\beta}([0, c], X)$. Hence, it follows from the generalized Banach contraction principle that $Q$ has one and only one fixed point $y$ in $C_{1-\beta}([0, c], X)$. This completes the proof.

\section{The Result of Approximate Controllability}

In this part, new sufficient conditions for the approximate controllability of the functional equation (2) are derived and proved by means of the iterative and approximate approach. For this purpose, we define operator $F: C_{1-\beta}([0$, $c], X) \rightarrow L^{p}([0, c], X)$ and a continuous linear mapping $G$ : $L^{p}([0, c], X) \rightarrow X$, respectively, by

$$
\begin{gathered}
F y(\tau)=g(\tau, y(\tau)), \quad \tau \in[0, c], \\
G \psi=\int_{0}^{c} S_{\beta}(c-\tau) \psi(\tau) d \tau,
\end{gathered}
$$

$$
\psi(\cdot) \in L^{p}([0, c], X)
$$

Let the pair $(y, u)$ be the mild solution of (2) with the control function $u \in V=L^{p}([0, c], U)$. We also denote the pair by $y(t)=y\left(t ; 0, y_{0}, u\right)$ and write the terminal state $y(c)$ by

$$
y(c)=y\left(c ; 0, y_{0}, u\right)=S_{\beta}(c) y_{0}+G(F y)+G(B u) .
$$

So the reachable set $K\left(c, y_{0}\right)$ of the functional equation (2) at time $c$ is

$$
K\left(c, y_{0}\right)=\left\{y_{c}: y_{c}=y\left(c ; 0, y_{0}, u\right) \text { for } u \in V\right\} .
$$

Thus the approximate controllability of functional equation (2) means the set $K\left(c, y_{0}\right)$ is dense on space $X$. In other words, we have the following expression of approximate controllability.

Definition 11. Let $y_{0} \in X$. We called the functional equation (2) approximately controllable on $[0, c]$ if, for any $\varepsilon>0$ and $y_{c} \in X$, there is a control function $u_{\varepsilon} \in V$ satisfying

$$
\left\|y_{c}-S_{\beta}(c) y_{0}-G\left(F y_{\varepsilon}\right)-G\left(B u_{\varepsilon}\right)\right\|<\varepsilon,
$$

where $y_{\varepsilon}(t)=y\left(t ; 0, y_{0}, u_{\varepsilon}\right), 0<t \leq c$.

Now, we give the following assumptions.

$\left(\mathrm{H}^{\prime}\right)$ There is a nonnegative number $L^{\prime}$ satisfying

$$
\|g(\tau, y)-g(\tau, z)\| \leq L^{\prime} \tau^{1-\beta}\|y-z\|
$$

for all $\tau \in[0, c]$ and $y, z \in X$.

(H4) For given $\varepsilon>0$ and $\psi \in L^{p}([0, c], X)$, there is a function $u \in V$ satisfying

$$
\begin{aligned}
\|G \psi-G(B u)\| & \leq \varepsilon, \\
\|B u\|_{L^{p}} & \leq \lambda\|\psi\|_{L^{p}},
\end{aligned}
$$

where $\lambda$ is a positive real number independent of $\psi$.

(H5) The following inequality holds:

$$
M L^{\prime} \lambda\left(\frac{p-1}{p \beta-1}\right)^{(p-1) / p} c E_{\beta}\left(M L^{\prime} c \Gamma(\beta)\right)<1,
$$

where $E_{\beta}(z)=\sum_{k=0}^{\infty}\left(z^{k} / \Gamma(k \beta+1)\right)$ is the Mittage-Leffler function and $M$ comes from Lemma 3 .

Because condition $\left(\mathrm{H}^{\prime}\right)$ implies $(\mathrm{H} 3)$, the existence result is still true if condition $(\mathrm{H} 3)$ is replaced by $\left(\mathrm{H}^{\prime}\right)$ in Theorem 10. Next, to prove the result of approximate controllability of functional equation (2), we need two lemmas below. 
Lemma 12. Let $\left(y_{1}, u_{1}\right),\left(y_{2}, u_{2}\right)$ be two pairs associated with the control system (2). Then under the hypotheses (H1), (H2), and $\left(\mathrm{H}^{\prime}\right)$ the following results hold:

$$
\begin{aligned}
& \left\|y\left(\cdot ; 0, y_{0}, u\right)\right\|_{C_{1-\beta}} \leq C E_{\beta}(M k c \Gamma(\beta)), \quad \forall u \in V, \\
& \left\|y_{1}-y_{2}\right\|_{C_{1-\beta}} \leq N E_{\beta}\left(M L^{\prime} c \Gamma(\beta)\right)\left\|B u_{1}-B u_{2}\right\|_{L^{p}},
\end{aligned}
$$

where $C=M\left[\left\|y_{0}\right\|+((p-1) /(p \beta-1))^{(p-1) / p} c^{1-1 / p}\left(\|B u\|_{L^{p}}+\right.\right.$ $\left.\left.\|\mu\|_{L^{p}}\right)\right], N=M((p-1) /(p \beta-1))^{(p-1) / p} c^{1-1 / p}$.

Proof. The mild solution $y(t)=y\left(t ; 0, y_{0}, u\right)$ of control system (2) in $C_{1-\beta}([0, c], X)$ satisfies

$$
\begin{aligned}
y(t)= & S_{\beta}(t) y_{0} \\
& +\int_{0}^{t} S_{\beta}(t-\tau)[B u(\tau)+g(\tau, y(\tau))] d \tau, \\
& 0<t \leq c .
\end{aligned}
$$

Thus, for $0 \leq t \leq c$, one has

$$
\begin{aligned}
& t^{1-\beta}\|y(t)\| \leq\left\|t^{1-\beta} S_{\beta}(t) y_{0}\right\|+t^{1-\beta} \int_{0}^{t}(t-\tau)^{\beta-1} \\
& \cdot\left\|(t-\tau)^{1-\beta} S_{\beta}(t-\tau) B u(\tau)\right\| d \tau \\
& +t^{1-\beta} \int_{0}^{t}(t-\tau)^{\beta-1} \\
& \cdot\left\|(t-\tau)^{1-\beta} S_{\beta}(t-\tau) g(\tau, y(\tau))\right\| d \tau \\
& \leq M\left[\left\|y_{0}\right\|+t^{1-\beta} \int_{0}^{t}(t-\tau)^{\beta-1}\|B u(\tau)\| d \tau\right. \\
& \left.+t^{1-\beta} \int_{0}^{t}(t-\tau)^{\beta-1}\left[\mu(\tau)+k \tau^{1-\beta}\|y(\tau)\|\right] d \tau\right] \\
& \leq M\left[\left\|y_{0}\right\|+\left(\frac{p-1}{p \beta-1}\right)^{(p-1) / p}\right. \\
& \cdot c^{1-1 / p}\left(\|B u\|_{L^{p}}+\|\mu\|_{L^{p}}\right) \\
& \left.+k c^{1-\beta} \int_{0}^{t}(t-\tau)^{\beta-1} \tau^{1-\beta}\|y(\tau)\| d \tau\right] .
\end{aligned}
$$

Set $\omega(t)=t^{1-\beta}\|y(t)\|, t \in[0, c]$. Thus,

$$
\omega(t) \leq C+M k c^{1-\beta} \int_{0}^{t}(t-\tau)^{\beta-1} \omega(\tau) d \tau
$$

By a generalized Gronwall inequality for convolution type integral equations (see Corollary 2 in [36]), we obtain

$$
\omega(t) \leq C E_{\beta}(M k c \Gamma(\beta)) .
$$

It follows that

$$
\|y\|_{C_{1-\beta}}=\sup _{t \in[0, c]} t^{1-\beta}\|y(t)\| \leq C E_{\beta}(M k c \Gamma(\beta)) .
$$

Now, let us take $y_{1}, y_{2} \in C_{1-\beta}([0, c], X)$ to be the mild solutions for the control system (2) with the control functions $u_{1}, u_{2} \in V$, respectively. Then, by the same way, we can get

$$
\left\|y_{1}-y_{2}\right\|_{C_{1-\beta}} \leq N E_{\beta}\left(M L^{\prime} c \Gamma(\beta)\right)\left\|B u_{1}-B u_{2}\right\|_{L^{p}} .
$$

This ends the proof.

Lemma 13. Under the condition (H1), for any $y \in D(A)$ there exists $\psi \in L^{p}([0, c], X)$ such that $G \psi=y$.

Proof. For $y \in D(A)$ and $\tau \in[0, c]$, let

$$
\begin{aligned}
& \psi_{1}(\tau)=\frac{\Gamma^{2}(\beta)}{c}(c-\tau)^{2(1-\beta)} S_{\beta}(c-\tau) y, \\
& \psi_{2}(\tau) \\
& =\frac{\Gamma^{2}(\beta)}{c} 2 \tau(c-\tau)^{1-\beta} \frac{d\left[(c-\tau)^{1-\beta} S_{\beta}(c-\tau) y\right]}{d \tau} .
\end{aligned}
$$

Then, we have

$$
\begin{aligned}
G \psi_{1} & =\frac{\Gamma^{2}(\beta)}{c} \int_{0}^{c} S_{\beta}(c-\tau)(c-\tau)^{2(1-\beta)} S_{\beta}(c-\tau) y d \tau \\
& =\frac{\Gamma^{2}(\beta)}{c} \int_{0}^{c}(c-\tau)^{2(1-\beta)} S_{\beta}^{2}(c-\tau) y d \tau \\
& =\left.\frac{\Gamma^{2}(\beta)}{c} \tau(c-\tau)^{2(1-\beta)} S_{\beta}^{2}(c-\tau) y\right|_{0} ^{c}-\frac{\Gamma^{2}(\beta)}{c} \\
& \cdot \int_{0}^{c} \tau d\left[(c-\tau)^{2(1-\beta)} S_{\beta}^{2}(c-\tau) y\right]=y-\frac{\Gamma^{2}(\beta)}{c} \\
\cdot & \int_{0}^{c} 2 \tau(c-\tau)^{1-\beta} S_{\beta}(c-\tau) d\left[(c-\tau)^{1-\beta} S_{\beta}(c-\tau) y\right] \\
= & y-G \psi_{2},
\end{aligned}
$$

which means that $G\left(\psi_{1}+\psi_{2}\right)=y$.

Moreover, it follows from the analyticity and the strong continuity of $\tau^{1-\beta} S_{\beta}(\tau)$ that $\psi_{1}+\psi_{2} \in L^{p}([0, c], X)$. This completes the proof.

Now, we can give the result about the approximate controllability of this section.

Theorem 14. Suppose conditions (H1), (H2), (H3'), (H4), and (H5) hold. Then the functional equation (2) is approximately controllable on the interval $[0, c]$.

Proof. Since $A$ is the infinitesimal generator of analytic $\beta$ order fractional resolvent of continuous operator $S_{\beta}(t)$ on space $X$, the domain of operator $A$ is dense in Banach space $X$. Thus, by the definition of approximate controllability, it suffices to prove that $D(A) \subset K\left(c, y_{0}\right)$. Next, we should prove that for any $\varepsilon>0$ and $y_{c} \in D(A)$, there is a control function $u_{\varepsilon} \in V$ with

$$
\left\|y_{c}-S_{\beta}(c) y_{0}-G\left(F y_{\varepsilon}\right)-G\left(B u_{\varepsilon}\right)\right\|<\varepsilon,
$$


where $y_{\varepsilon}(t)=y\left(t ; 0, y_{0}, u_{\varepsilon}\right)$ satisfies

$$
\begin{aligned}
y_{\varepsilon}(t)= & S_{\beta}(t) y_{0}+\int_{0}^{t} S_{\beta}(t-\tau) F y_{\varepsilon}(\tau) d \tau \\
& +\int_{0}^{t} S_{\beta}(t-\tau) B u_{\varepsilon}(\tau) d \tau, \quad 0<t \leq c .
\end{aligned}
$$

It follows from the analyticity of fractional resolvent that $S_{\beta}(c) y_{0} \in D(A)$ for $y_{0} \in X$, which implies that $y_{c}-S_{\beta}(c) y_{0} \in$ $D(A)$ for $y_{c} \in D(A)$. Then, by Lemma 13 there is a function $\psi \in L^{p}([0, c], X)$ satisfying

$$
G \psi=y_{c}-S_{\beta}(c) y_{0} .
$$

Thus, for every $\varepsilon>0$ and $u_{1} \in V$, by means of hypothesis (H4), we can find a function $u_{2} \in V$ satisfying

$$
\left\|y_{c}-S_{\beta}(c) y_{0}-G\left(F y_{1}\right)-G\left(B u_{2}\right)\right\|<\frac{\varepsilon}{3^{2}} \text {, }
$$

where $y_{1}(t)=y\left(t ; 0, y_{0}, u_{1}\right), 0<t \leq c$. Further, for $u_{2} \in V$, we determine again $v_{2} \in V$ by condition (H4) and Lemma 12 with the following two properties:

$$
\begin{aligned}
& \left\|G\left(F y_{2}-F y_{1}\right)-G\left(B v_{2}\right)\right\|<\frac{\varepsilon}{3^{3}} \\
& \left\|B v_{2}\right\|_{L^{p}} \leq \lambda\left\|F y_{1}-F y_{2}\right\|_{L^{p}} \leq \lambda L^{\prime} c^{1 / p}\left\|y_{2}-y_{1}\right\|_{C_{1-\beta}} \\
& \leq \lambda L^{\prime} M\left(\frac{p-1}{p \beta-1}\right)^{(p-1) / p} c E_{\beta}\left(M L^{\prime} c \Gamma(\beta)\right) \\
& \cdot\left\|B u_{1}-B u_{2}\right\|_{L^{p}},
\end{aligned}
$$

where $y_{2}(t)=y\left(t ; 0, y_{0}, u_{2}\right), 0<t \leq c$.

Thus, we may define $u_{3}=u_{2}-v_{2}$ in $V$, which has the following property

$$
\begin{aligned}
\| y_{c}- & S_{\beta}(c) y_{0}-G\left(F y_{2}\right)-G\left(B u_{3}\right) \| \\
\leq & \left\|y_{c}-S_{\beta}(c) y_{0}-G\left(F y_{1}\right)-G\left(B u_{2}\right)\right\| \\
& +\left\|G\left(B v_{2}\right)-G\left(F y_{2}-F y_{1}\right)\right\| \leq\left(\frac{1}{3^{2}}+\frac{1}{3^{3}}\right) \varepsilon .
\end{aligned}
$$

By the same way, we obtain the sequence $\left\{u_{n}: n \geq 1\right\} \subseteq V$ satisfying

$$
\begin{aligned}
& \left\|y_{c}-S_{\beta}(c) y_{0}-G\left(F y_{n}\right)-G\left(B u_{n+1}\right)\right\| \\
& \leq\left(\frac{1}{3^{2}}+\cdots+\frac{1}{3^{n+1}}\right) \varepsilon, \\
& \left\|B u_{n+1}-B u_{n}\right\|_{L^{p}} \leq \lambda L^{\prime} M\left(\frac{p-1}{p \beta-1}\right)^{(p-1) / p} \\
& \cdot c E_{\beta}\left(M L^{\prime} c \Gamma(\beta)\right)\left\|B u_{n}-B u_{n-1}\right\|_{L^{p}},
\end{aligned}
$$

where $y_{n}(t)=y\left(t ; 0, y_{0}, u_{n}\right), 0<t \leq c, n=1,2, \ldots$. Since the condition (H5) is satisfied, it follows that the sequence
$\left\{B u_{n}: n \geq 1\right\}$ is a Cauchy sequence in $L^{p}([0, c], X)$ and thus we can obtain a function $v \in L^{p}([0, c], X)$ with

$$
\lim _{n \rightarrow \infty} B u_{n}=v \quad \text { in } L^{p}([0, c], X) .
$$

Note that the mapping $G: L^{p}([0, c], X) \rightarrow X$ is a continuous linear operator. Therefore for every $\varepsilon>0$, we can find a real integer number $N_{\varepsilon}>0$ satisfying

$$
\left\|G\left(B u_{N_{\varepsilon}+1}\right)-G\left(B u_{N_{\varepsilon}}\right)\right\|<\frac{\varepsilon}{3} .
$$

Consequently, by inequalities (43) and (46) we derive

$$
\begin{aligned}
\| y_{c} & -S_{\beta}(c) y_{0}-G\left(F y_{N_{\varepsilon}}\right)-G\left(B u_{N_{\varepsilon}}\right) \| \\
\leq & \left\|y_{c}-S_{\beta}(c) y_{0}-G\left(F y_{N_{\varepsilon}}\right)-G\left(B u_{N_{\varepsilon}+1}\right)\right\| \\
& \quad+\left\|G\left(B u_{N_{\varepsilon}+1}\right)-G\left(B u_{N_{\varepsilon}}\right)\right\| \\
\leq & \left(\frac{1}{3^{2}}+\cdots+\frac{1}{3^{N_{\varepsilon}+1}}\right) \varepsilon+\frac{\varepsilon}{3} \leq \varepsilon,
\end{aligned}
$$

where $y_{N_{\varepsilon}}(t)=y\left(t ; 0, y_{0}, u_{N_{\varepsilon}}\right), 0<t \leq c$. This ends the proof.

Remark 15. In this article, we consider the control system with Riemann-Liouville derivative under the general assumption that linear operator $A: D(A) \subset X \rightarrow X$ generates a fractional resolvent $S_{\beta}(t), t>0$ on the Banach space $X$. By the resolvent theory, fixed point theorem, and iterative and approximate approach, we obtain the results of existence and uniqueness for mild solutions and approximate controllability of functional control system. From this point of view, our results extend the theorems in $[17,18]$, where the authors assumed that the operator $A$ generates $C_{0}$-semigroups. As for examples, we refer the reader to $[17,18,33]$ since the examples therein still hold in our framework.

\section{Conflicts of Interest}

The authors declare that there are no conflicts of interest regarding the publication of this paper.

\section{Acknowledgments}

The work was supported by Special Fund for Public Welfare Research of Ministry of Land and Resources of China (Grant no. 201411007).

\section{References}

[1] T. E. Duncan, Y. Hu, and B. Pasik-Duncan, "Stochastic calculus for fractional Brownian motion. I. Theory," SIAM Journal on Control and Optimization, vol. 38, no. 2, pp. 582-612, 2000.

[2] R. Hilfer, Applications of Fractional Calculus in Physics, World Scientific, Singapore, 2000.

[3] I. Podlubny, Fractional Differential Equations, vol. 198 of Mathematics in Science and Engineering, Academic Press, San Diego, Calif, USA, 1999. 
[4] V. E. Tarasov, Fractional Dynamics: Application of Fractional Calculus to Dynamics of Particles, Fields and Media, Springer, New York, NY, USA, 2010.

[5] Y. Wang and L. Liu, "Uniqueness and existence of positive solutions for the fractional integro-differential equation," Boundary Value Problems, vol. 12, pp. 1-17, 2017.

[6] X. Zhang, L. Liu, Y. Wu, and B. Wiwatanapataphee, "Nontrivial solutions for a fractional advection dispersion equation in anomalous diffusion," Applied Mathematics Letters, vol. 66, pp. $1-8,2017$.

[7] X. Zhang, Y. Wu, and L. Caccetta, "Nonlocal fractional order differential equations with changing-sign singular perturbation," Applied Mathematical Modelling, vol. 39, no. 21, pp. 65436552, 2015.

[8] X. G. Zhang, L. S. Liu, Y. H. Wu, and B. Wiwatanapataphee, “The spectral analysis for a singular fractional differential equation with a signed measure," Applied Mathematics and Computation, vol. 257, pp. 252-263, 2015.

[9] X. Zhang, L. Liu, and Y. Wu, "The uniqueness of positive solution for a fractional order model of turbulent flow in a porous medium," Applied Mathematics Letters, vol. 37, pp. 26-33, 2014.

[10] Z. Fan, "Approximate controllability of fractional differential equations via resolvent operators," Advances in Difference Equations, vol. 54, pp. 1-11, 2014.

[11] Z. Fan and G. M. Mophou, "Remarks on the controllability of fractional differential equations," Optimization, vol. 63, no. 8, pp. 1205-1217, 2014.

[12] Z. Fan, Q. Dong, and G. Li, "Approximate controllability for semilinear composite fractional relaxation equations," Fractional Calculus and Applied Analysis, vol. 19, no. 1, pp. 267-284, 2016.

[13] S. Kumar and N. Sukavanam, "Approximate controllability of fractional order neutral control systems with delay," International Journal of Nonlinear Science, vol. 13, no. 4, pp. 454-462, 2012.

[14] S. Kumar and N. Sukavanam, "Approximate controllability of fractional order semilinear systems with bounded delay," Journal of Differential Equations, vol. 252, no. 11, pp. 6163-6174, 2012.

[15] J. Liang and H. Yang, "Controllability of fractional integrodifferential evolution equations with nonlocal conditions," Applied Mathematics and Computation, vol. 254, pp. 20-29, 2015.

[16] F. Li, J. Liang, and H.-K. Xu, "Existence of mild solutions for fractional integrodifferential equations of Sobolev type with nonlocal conditions," Journal of Mathematical Analysis and Applications, vol. 391, no. 2, pp. 510-525, 2012.

[17] Z. Liu and X. Li, "Approximate controllability of fractional evolution systems with Riemann-Liouville fractional derivatives," SIAM Journal on Control and Optimization, vol. 53, no. 4, pp. 1920-1933, 2015.

[18] Z. Liu and M. Bin, "Approximate controllability of impulsive Riemann-Liouville fractional equations in Banach spaces," Journal of Integral Equations and Applications, vol. 26, no. 4, pp. 527551, 2014.

[19] C. Lizama, "Regularized solutions for abstract Volterra equations," Journal of Mathematical Analysis and Applications, vol. 243, no. 2, pp. 278-292, 2000.

[20] N. I. Mahmudov, "Approximate controllability of semilinear deterministic and stochastic evolution equations in abstract spaces," SIAM Journal on Control and Optimization, vol. 42, no. 5, pp. 1604-1622, 2003.

[21] N. I. Mahmudov, "Approximate controllability of evolution systems with nonlocal conditions," Nonlinear Analysis. Theory, Methods \& Applications, vol. 68, no. 3, pp. 536-546, 2008.

[22] N. I. Mahmudov, "Approximate controllability of fractional Sobolev-type evolution equations in Banach spaces," Abstract and Applied Analysis, vol. 2013, Article ID 502839, 9 pages, 2013.

[23] G. Mophou and G. M. N'Guérékata, "Optimal control of a fractional diffusion equation with state constraints," Computers \& Mathematics with Applications, vol. 62, no. 3, pp. 1413-1426, 2011.

[24] G. M. Mophou, "Optimal control of fractional diffusion equation," Computers \& Mathematics with Applications, vol. 61, no. 1, pp. 68-78, 2011.

[25] R. Sakthivel, Y. Ren, and N. I. Mahmudov, "Approximate controllability of second-order stochastic differential equations with impulsive effects," Modern Physics Letters B, vol. 24, no. 14, pp. 1559-1572, 2010.

[26] R. Sakthivel, J. J. Nieto, and N. I. Mahmudov, "Approximate controllability of nonlinear deterministic and stochastic systems with unbounded delay," Taiwanese Journal of Mathematics, vol. 14, no. 5, pp. 1777-1797, 2010.

[27] P. Tamilalagan and P. Balasubramaniam, "Approximate controllability of fractional stochastic differential equations driven by mixed fractional Brownian motion via resolvent operators," International Journal of Control, vol. 90, no. 8, pp. 1713-1727, 2017.

[28] H. X. Zhou, "Approximate controllability for a class of semilinear abstract equations," SIAM Journal on Control and Optimization, vol. 21, no. 4, pp. 551-565, 1983.

[29] H. Oka, "Linear Volterra equations and integrated solution families," Semigroup Forum, vol. 53, no. 3, pp. 278-297, 1996.

[30] M. Li, Q. Zheng, and J. Zhang, "Regularized resolvent families," Taiwanese Journal of Mathematics, vol. 11, no. 1, pp. 117-133, 2007.

[31] J. Wang and Y. Zhou, "A class of fractional evolution equations and optimal controls," Nonlinear Analysis. Real World Applications, vol. 12, no. 1, pp. 262-272, 2011.

[32] K. Li and J. Peng, "Fractional resolvents and fractional evolution equations," Applied Mathematics Letters, vol. 25, no. 5, pp. 808812, 2012.

[33] Z. Fan, "Existence and regularity of solutions for evolution equations with Riemann-Liouville fractional derivatives," Indagationes Mathematicae, vol. 25, no. 3, pp. 516-524, 2014.

[34] A. A. Kilbas, H. M. Srivastava, and J. J. Trujillo, Theory and Applications of Fractional Differential Equations, vol. 204 of North-Holland Mathematics Studies, Elsevier Science, Amsterdam, Netherlands, 2006.

[35] W. Arendt, C. J. K. Batty, M. Hieber, and F. Neubrander, VectorValued Laplace Transforms and Cauchy Problems, vol. 96, Birkhäuser, Basel, Switzerland, 2001.

[36] H. Ye, J. Gao, and Y. Ding, "A generalized Gronwall inequality and its application to a fractional differential equation," Journal of Mathematical Analysis and Applications, vol. 328, no. 2, pp. 1075-1081, 2007. 


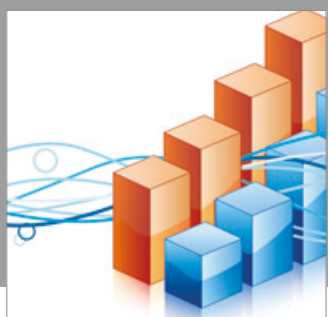

Advances in

Operations Research

vatersals

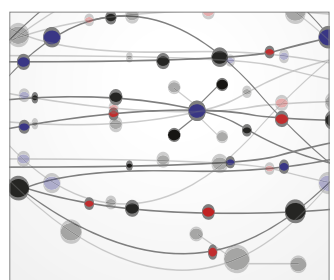

\section{The Scientific} World Journal
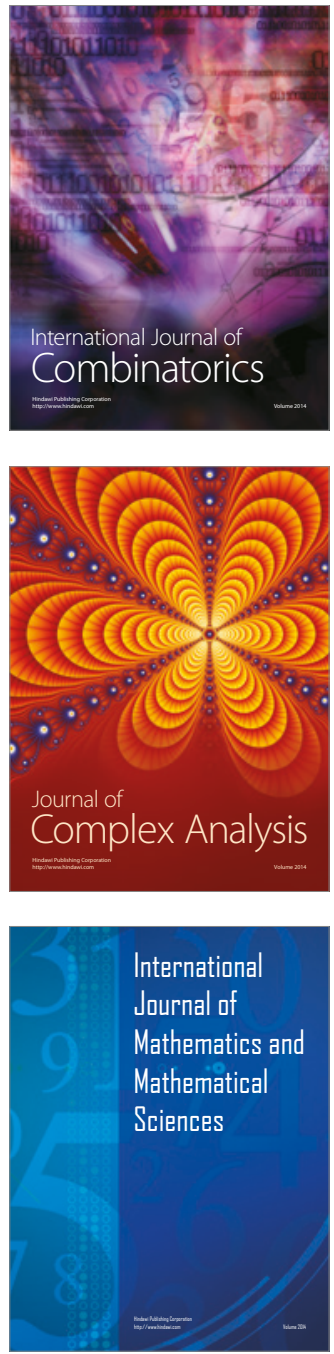
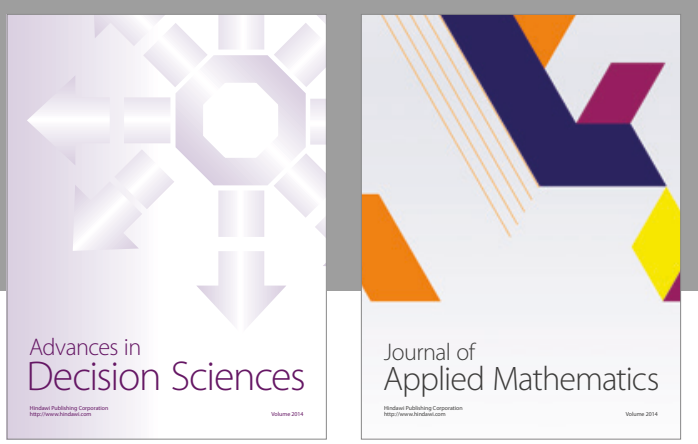

Algebra

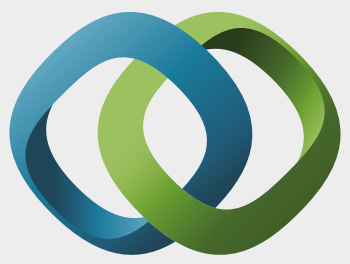

\section{Hindawi}

Submit your manuscripts at

https://www.hindawi.com
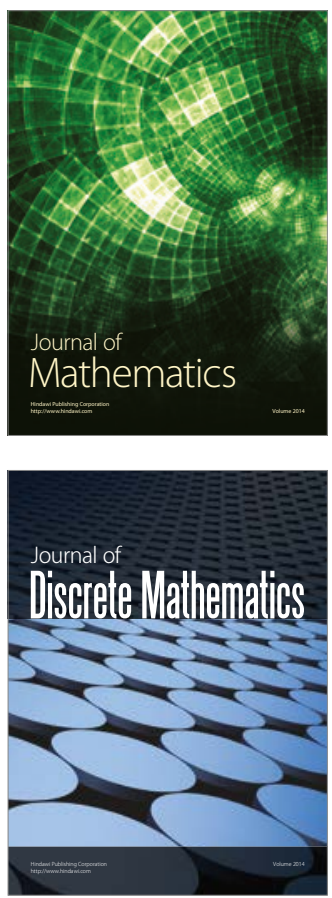

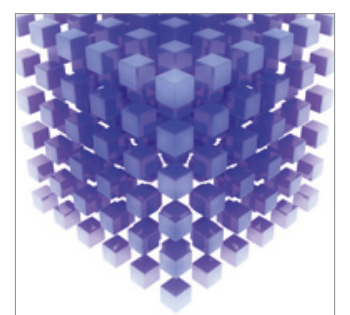

Mathematical Problems in Engineering
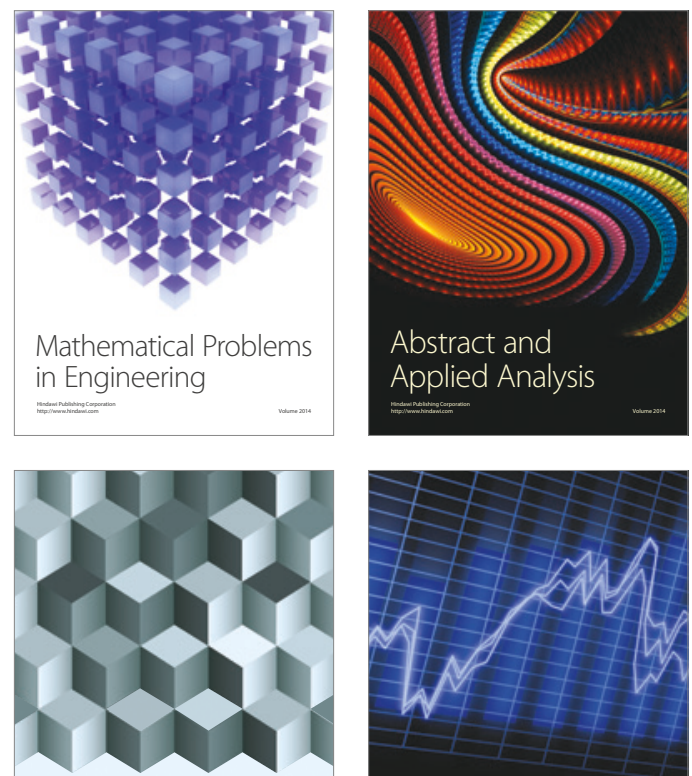

Journal of

Function Spaces

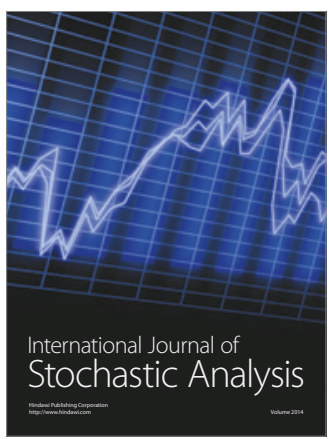

Probability and Statistics
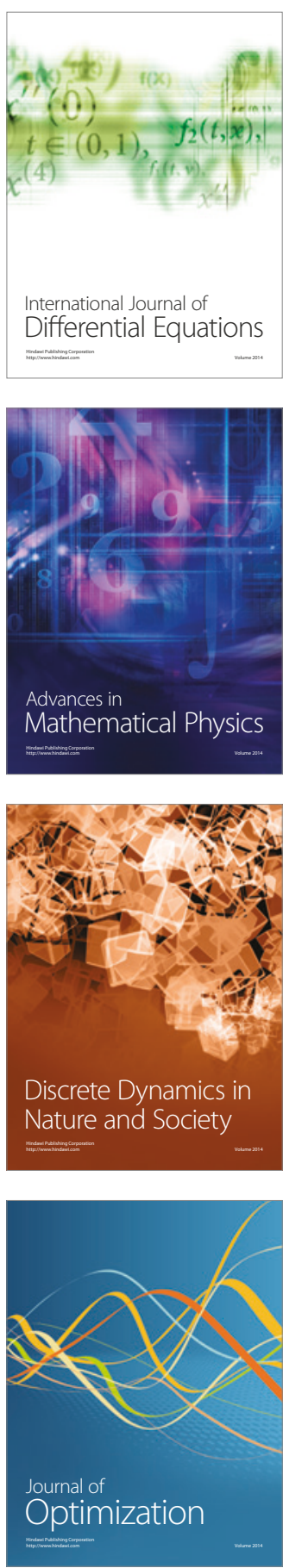